\title{
Current opinion in the molecular genetics of Adams-Oliver syndrome
}

Laura Southgate

\section{Abstract:}

Introduction: Adams-Oliver syndrome (AOS) is an orphan disorder of terminal transverse limb defects (TTLD) and aplasia cutis congenita (ACC), hypothesized to occur as a consequence of disordered vasculogenesis. AOS is estimated to affect 1 in 225,000 live births, however comprehensive studies of geographical prevalence remain limited. Areas covered: This review summarises current opinion in the molecular genetics of AOS and provides recommended updates to the diagnostic criteria. Our understanding of the clinical features associated with AOS has been much improved due to recent advancement in establishing the underlying genetic causes of the condition. To date, six causal genes have been described, which together specifically implicate Rho GTPase dysregulation and perturbed Notch signalling as central to disease development. Despite these genetic advances, mutations in the established genes only represent $36 \%$ of reported AOS cases, indicating a large degree of missing heritability still to be resolved. Furthermore, the fundamental mechanisms underlying AOS remain undefined, impeding diagnostic and treatment progression. Expert Opinion: Further work to examine structural variation and identify novel genes is necessary to explain the missing heritability in AOS. In the future, dedicated functional analyses will be required to delineate the pathogenic mechanisms and facilitate focussed evaluation of targeted therapies.

\section{Keywords:}

Adams-Oliver syndrome; Molecular genetics; Notch signalling; Rho GTPase regulation; Vascular development

\section{Main text:}

\subsection{Clinical features and diagnosis:}

First described by Forrest H. Adams and Clarence P. Oliver in 1945 as "arrested development" of the extremities and skull, AOS is distinct from congenital amputations due to its Mendelian inheritance pattern and association with a characteristic focal absence of epidermis on the scalp vertex.[1] The family in this original case report epitomises an important feature of the condition, namely variable expression of the phenotype within families. TTLD is typically asymmetrical and can range in severity from mild digit shortening to complete absence of the hands or feet. Brachydactyly, syndactyly and hemimelia have also been documented.[1,2] Similarly, ACC varies in diameter from $<1$ to $>10 \mathrm{~cm}$ and can occur with or without an underlying skull defect. Both TTLD and ACC may manifest as isolated defects and a complete absence of clinical features in obligate gene carriers supports reduced 
penetrance of the disease allele, representing a diagnostic challenge. Given such variability in phenotypic expression, the following criteria are recommended as compatible with a diagnosis of AOS: a) clinical findings of a combination of scalp ACC and TTLD; b) ACC and/or TTLD and an affected first-degree relative; c) ACC and/or TTLD in conjunction with identified mutation in a recognised AOS-related gene (Table 1).[2]

AOS is associated with numerous associated features, broadly comprising cardiovascular defects, neurological abnormalities, ocular anomalies and other developmental disorders. [3,4] Congenital heart defects (CHDs) are detected in $23 \%$ of cases and represent significant morbidity and mortality. $[2,5]$ AOS-related CHDs cover a wide range of severity, predominantly encompassing leftsided obstructive defects, and are often associated with vascular abnormalities, the most frequent of which is cutis marmorata telangiectatica congenita (CMTC). Other vascular defects may impact the lower limb vasculature, portal and cortical renal veins, or the pulmonary vasculature.[4,6] Importantly, the association between cardiovascular defects and isolated ACC or TTLD has been demonstrated to be aetiologically related to AOS.[7]

Abnormalities of the central nervous system (CNS) and structural eye anomalies, for example microphthalmia and retinal vasculopathy, appear more commonly associated with autosomal recessive inheritance. $[2,5,8]$ Developmental delay and seizures each occur in $8-9 \%$ of AOS cases overall, but are significantly more frequent $(>45 \%)$ in patients with microcephaly and are strongly correlated with intracranial vascular defects.[5,9] Additional developmental defects manifesting in $<2 \%$ of cases include gastrointestinal, renal or reproductive anomalies and facial dysmorphism. Based on the prevalence of cardiovascular and neurological abnormalities in AOS, the following additional diagnostic criteria should be considered highly suggestive of a diagnosis: a) ACC and/or TTLD in combination with CHD, CMTC or other vascular anomaly; b) ACC and/or TTLD in association with CNS defects; c) cardiovascular or CNS defects and a first-degree relative with features consistent with AOS (Table 1).

\subsection{Molecular genetics of AOS:}

The first causal AOS gene was identified in 2011 by genome-wide analysis in two families with ACC and TTLD co-segregating with autosomal dominant inheritance. Following detection of a single linkage peak, heterozygous variants of the Rho GTPase-activating protein 31 (ARHGAP31) gene were isolated by candidate gene analysis and targeted exome sequencing.[10] ARHGAP31 dysregulation accounts for a relatively small proportion (2\%) of independent AOS cases. Whilst limited conclusions can be drawn from the small number reported to date, all ARHGAP31 mutations are proteintruncating variants located in the terminal exon 12, of which c.2047C >T (p.GIn683*) is recurrent.[9] Mutations of ARHGAP31 are highly correlated with TTLD specifically (Table 2 ) and, thus far, no cardiovascular or neurological defects have been described in this cohort.[11] Subsequent discovery of homozygous and compound heterozygous mutations in the dedicator of cytokinesis 6 (DOCK6) gene confirmed the existence of an autosomal recessive form of AOS and cemented the central role of Rho GTPase dysregulation in AOS pathogenesis.[12] Identified DOCK6 variants cover all mutation categories, including missense and splicing variants, and represent $7 \%$ of reported AOS cases. Whilst the vast majority exhibit the hallmark features of AOS, DOCK6 mutation carriers also have a broad range of additional abnormalities and are strongly correlated with CNS and ocular defects (Table 
2). $[8,9]$ Of note, it has been demonstrated that DOCK6 harbours a high than expected frequency of likely deleterious variation in the general population, highlighting the possibility of a more complex disease aetiology for this gene, potentially dependent on genetic background.[13]

More recently, a particular focus has developed around the Notch signalling cascade in the aetiology of AOS. In 2012, whole-exome sequencing of two autosomal dominant AOS kindreds revealed heterozygous mutations of the recombination signal binding protein for immunoglobulin kappa J region (RBPJ) gene.[14] RBPJ mutations are a relatively rare cause of AOS and appear to underlie a classical form of the condition, predominantly characterised by TTLD and ACC (Table 2). By contrast, mutations in the epidermal growth factor (EGF) domain-specific O-linked $\mathrm{N}$-acetylglucosamine transferase (EOGT) gene are potentially associated with an autosomal recessive form of ACC and syndactyly (Table 2). EOGT was identified as a causal AOS gene through genome-wide autozygosity mapping and has been independently validated in a small number of sporadic cases. $[9,15]$ of interest, nine probands share a c.1074delA (p.Gly359Aspfs*28) mutation; however, as the majority belong to Bedouin tribes, this likely indicates a founder mutation in the Arab population.[15,16]

The major genetic risk factors for autosomal dominant AOS are NOTCH1 and the delta-like canonical Notch ligand 4 (DLL4). Mutations in NOTCH1 represent one third of all reported AOS variants (11\% of published case reports) and are associated with an over-representation of CHDs by comparison to the wider AOS cohort. $[7,17]$ NOTCH1 missense variation is common in AOS and some mutation carriers exhibit incomplete penetrance. Consequently, $>20 \%$ of identified $\mathrm{NOTCH} 1$ variants are considered variants of unknown significance (and have therefore not been included here).[9] Given the clustering of AOS mutations within the Notch pathway, DLL4 was screened as a candidate gene due to its essential role in vascular development. Missense or nonsense DLL4 mutations account for $\sim 6 \%$ of all reported AOS cases and, by contrast to ARHGAP31, have a stronger correlation with ACC (Table 2).[9,18]

\subsection{Rho GTPase dysregulation:}

The Rho GTPases are key regulators of various cellular activities including actin cytoskeletal reorganization and microtubule dynamics. Through a molecular switch mechanism, they cycle between two conformations - active (GTP-bound) and inactive (GDP-bound) - regulated by the actions of guanosine nucleotide-exchange factors (GEFs) and GTPase-activating proteins (GAPs). As Rho GTPase regulators, ARHGAP31 and the GEF DOCK6 have opposing effects on the small GTPases Rac1 and Cdc42, which are important in the control of cell migration through promoting lamellipodia and filopodia formation, respectively.[19-21] Both Arhgap31 and Dock6 display localised expression to the surface ectoderm, limb buds and heart during mouse development, demonstrating a clear correlation with the sites of phenotypic expression in AOS.[10,12] Analysis of patient-derived fibroblasts carrying ARHGAP31 mutation has revealed cellular defects consistent with Cdc42/Rac1 dysregulation, including impaired cell migration and cell rounding.[10] ARHGAP31 has been demonstrated to modulate directed and random cell migration by influencing adhesion dynamics, suggesting that the molecular mechanism in AOS may be a disruption of cell adhesion contacts. $[20,22]$ More recently, analysis of a knock-out mouse model has highlighted a critical role for Arhgap31 in vascular development and VEGF-mediated angiogenesis, providing a novel molecular link between Rho GTPase dysregulation and vascular development in this disorder.[23] 
These findings further support the hypothesis that AOS represents a constellation of clinical features resulting from an early embryonic vascular abnormality.[24]

ARHGAP31 mutant transcripts are stable and have been demonstrated to lead to constitutive inactivation of $\mathrm{Cdc} 42$ through a gain-of-function mechanism, potentially due to protein truncation impeding regulation of the RhoGAP domain.[10] Complementary loss-of-function mutations in DOCK6 support a model of AOS caused by the abnormal accumulation of inactive Cdc42/Rac1. A recent example of cell-intrinsic adaptation indicates that alternative $\mathrm{Cdc42/Rac1} \mathrm{regulatory}$ mechanisms may compensate for loss of DOCK6 during development.[25] This phenomenon appears to be modulated through down-regulation of the ubiquitin-like modifier, interferon stimulated gene 15 (ISG15).[26] A further study has demonstrated a link between DOCK6 and the transforming growth factor beta (TGF- $\beta$ ) signalling cascade, wherein induction of microRNA-142-3p by the TGF- $\beta$ pathway leads to a down-regulation of DOCK6.[27] This DOCK6 repression appears to inhibit cell migration in vascular smooth muscle cells, providing preliminary evidence of a role for DOCK6 dysregulation during vascular development in AOS.

\subsection{Notch signalling in AOS:}

The highly conserved Notch pathway is known to be critical for many developmental processes. The canonical Notch cascade is activated by extracellular binding of a delta-like (DLL) or jagged (JAG) ligand from an adjacent cell, triggering cleavage of the Notch intracellular domain (NICD). NICD subsequently translocates to the nucleus, where it complexes with the transcription factor RBPJ to regulate the expression of Notch target genes, for example HES1 and HEY1. In AOS, missense mutations within the DNA-binding domain of RBPJ have been shown to attenuate its binding to the HES1 promoter, leading to dysregulated Notch signalling.[14] Similarly NOTCH1 mutation-positive patients have reduced $\mathrm{NOTCH} 1$ expression with an associated decrease in transcript abundance of HES1 and HEY1.[7] Of interest, ARHGAP31 has recently been discovered to exhibit transcriptional activity, which is absent in the recurrent p.GInQ683* nonsense variant, leading to the possibility of potential crosstalk between ARHGAP31 and the Notch signalling pathway.[28]

The NOTCH1 extracellular domain is composed of 36 EGF-like repeats, which are modified by posttranslational glycosylation with $O$-glycan residues. Examination of glycosyltransferase activity in mutant EOGT proteins in vitro has suggested that AOS may be caused by defective $O$-GlcNAcylation in the endoplasmic reticulum. [29] EOGT regulates Notch signalling through $O$-linked $N$ acetylglucosamine (O-GlcNAc) modification and it has been demonstrated that EOGT-deficient cells have a decreased binding affinity for DLL4, likely due to impaired glycosyltransferase activity on key EGF repeats of NOTCH1.[30] Whilst NOTCH1 variants have also been reported to cause nonsyndromic CHDs, highlighting the critical role of Notch signalling during cardiovascular development, mutations detected in AOS patients represent a distinct allelic series of novel NOTCH1 variation, with evidence of unique clustering around the ligand-binding domain, specified by EGF repeats 11-13. Key variation within this domain comprises cysteine substitutions likely to disrupt disulphide bonds.[7, 9] The subsequent identification of novel missense mutations within the reciprocal Notch-binding region of the DLL4 ligand now point to a crucial role for the NOTCH1/DLL4 receptor-ligand complex in AOS pathogenesis.[18] 


\section{Conclusion:}

Genetic studies elucidating the molecular aetiology of AOS have provided a unique window into key cellular pathways important during human vascular development. While preliminary analyses offer a tantalising glimpse into the molecular mechanisms involved in AOS pathogenesis, a comprehensive investigation of the functional consequences of identified variants is now required to ascertain the respective roles of Rho and Notch pathways in the aetiology of this heterogeneous disorder.

\section{Expert opinion:}

Investigating rare genetic disorders such as AOS remains of value but carries inherent difficulties in establishing sample sizes with sufficient power for novel gene detection, particularly in the context of phenotypic and locus heterogeneity. Whilst diagnosis remains challenging, the development of next-generation sequencing has facilitated the rapid detection of causal genes, leading to improved clinical management for patients and their families. The identification of six AOS genes over the past few years now provides the possibility of a molecular diagnosis in at least one third of cases, with important opportunities for genetic counselling and prenatal testing, where applicable.

Although important insights into the key cellular processes involved in AOS pathogenesis have been gleaned from genetic studies, major focus must still be given to expansion of the genetic architecture through both the identification of novel genes and systematic mutation screening of the established gene loci. Previous reports have suggested that partial gene deletions or duplications can account for $10-30 \%$ of disease-causing mutations in some disorders,[31] indicating that a targeted analysis of copy number variation (CNV) in AOS may explain a significant proportion of missing heritability. Indeed, a de novo genomic deletion across the 5 ' region of NOTCH1 has previously been reported in AOS,[17] and CNV screening has successfully identified the underlying genetic aetiology for other major limb reduction malformations.[32] Aligned to this is the necessity for comprehensive clinical phenotyping to support investigations of genotype-phenotype correlations and the isolation of disease genes underpinning homogeneous cohorts. Detailed radiological imaging, cardiac and neurological examination should therefore be performed for all patients with a suggested diagnosis of AOS. In the absence of clinical and genetic screening in all reported cases, estimates of the relative proportions of AOS-associated defects and number of patients having achieved a molecular diagnosis remain limited.

Undoubtedly, elucidating the genetic aetiology is just the first step in understanding the development and clinical progression of AOS. Nonetheless, genetic studies have highlighted the key pathways involved, laying the groundwork for the development of directed assays to evaluate Rho GTPase dysregulation and impaired Notch signalling during vasculogenesis. Further examination of the EGF domains, and in particular the ligand-binding region, of NOTCH1 is particularly enticing given the clear link between EOGT glycosylation and DLL4-mediated Notch signalling. Of interest, DII4mediated Notch signalling suppresses endothelial tip cell differentiation and filopodia extension during vascular development, providing a potential mechanistic link between the Notch pathway and Cdc42 inactivation.[33] It remains to be seen whether these ostensibly disparate pathways can be brought together into an integrated network for targeted drug discovery. Until then, therapy 
options remain limited and patient management will be reliant on careful monitoring and prompt treatment of major manifestations, rather than prevention.

\section{Article highlights box:}

- Adams-Oliver syndrome is a clinically and genetically heterogeneous disorder

- Familial segregation supports both autosomal dominant and autosomal recessive inheritance, with sporadic cases representing around one third

- To date, six causal genes have been identified, accounting for $36 \%$ of reported AOS cases

- Rho GTPase dysregulation and perturbed Notch signalling are central to disease development

- The co-existence of cardiovascular features indicates disrupted vasculogenesis as the primary pathogenic mechanism, yet this remains to be fully explored

\section{Bibliography:}

\section{* = of interest}

** $=$ of considerable interest

1. Adams FH, Oliver CP. Hereditary deformities in man due to arrested development. J Hered. 1945;36:3-7.

2. Lehman A, Wuyts W, Patel MS. Adams-Oliver Syndrome. In: Adam MP, Ardinger HH, Pagon RA, et al. (eds.) GeneReviews [Internet]. Seattle (WA): University of Washington, Seattle. April 2016.

3. Snape KM, Ruddy D, Zenker M, et al. The spectra of clinical phenotypes in aplasia cutis congenita and terminal transverse limb defects. Am J Med Genet A. 2009;149A(8):1860-1881.

4. Southgate L, Trembath RC (June 2016). Chapter 183: ARHGAP31, DOCK6, RBPJ and EOGT and Adams-Oliver Syndrome. In: CJ Epstein, RP Erickson, A Wynshaw-Boris (eds.) Inborn Errors of Development: The Molecular Basis of Clinical Disorders of Morphogenesis. 3rd edition. New York: Oxford University Press, pp.1203-1209.

5. Hassed S, Li S, Mulvihill J, Aston C, Palmer S. Adams-Oliver syndrome review of the literature: Refining the diagnostic phenotype. Am J Med Genet A. 2017;173(3):790-800

6. Digilio MC, Marino B, Baban A, Dallapiccola B. Cardiovascular malformations in Adams-Oliver syndrome. Am J Med Genet A. 2015;167A(5):1175-1177.

7. Southgate L, Sukalo M, Karountzos AS, et al. Haploinsufficiency of the NOTCH1 receptor as a cause of Adams-Oliver syndrome with variable cardiac anomalies. Circ Cardiovasc Genet. 2015;8(4):572-581.

8. Sukalo M, Tilsen F, Kayserili $\mathrm{H}$, et al. DOCK6 mutations are responsible for a distinct autosomalrecessive variant of Adams-Oliver syndrome associated with brain and eye anomalies. Hum Mutat. 2015;36(11):1112. 
9. Meester JAN, Sukalo M, Schröder KC, et al. Elucidating the genetic architecture of Adams-Oliver syndrome in a large European cohort. Hum Mutat. 2018;39(9):1246-1261.

**A comprehensive mutational analysis of a large cohort of AOS patients, which re-examines the likely pathogenicity of variants described to date, highlighting important genotype-phenotype correlations and emphasizing the missing heritability in this condition.

10. Southgate L, Machado RD, Snape KM, et al. Gain-of-function mutations of ARHGAP31, a Cdc42/Rac1 GTPase regulator, cause syndromic cutis aplasia and limb anomalies. Am J Hum Genet. 2011;88(5):574-585.

11. Isrie $M$, Wuyts $W$, Van Esch $H$, Devriendt K. Isolated terminal limb reduction defects: extending the clinical spectrum of Adams-Oliver syndrome and ARHGAP31 mutations. Am J Med Genet A. 2014;164A(6):1576-1579.

12. Shaheen $R$, Faqeih $E$, Sunker $A$, et al. Recessive mutations in DOCK6, encoding the guanidine nucleotide exchange factor DOCK6, lead to abnormal actin cytoskeleton organization and Adams-Oliver syndrome. Am J Hum Genet. 2011;89(2):328-333.

13. Lehman A, Stittrich AB, Glusman G. Diffuse angiopathy in Adams-Oliver syndrome associated with truncating DOCK6 mutations. Am J Med Genet A. 2014;164A(10):2656-2662.

14. Hassed SJ, Wiley GB, Wang S, et al. RBPJ mutations identified in two families affected by AdamsOliver syndrome. Am J Hum Genet. 2012;91(2):391-395.

15. Shaheen R, Aglan M, Keppler-Noreuil K, et al. Mutations in EOGT confirm the genetic heterogeneity of autosomal-recessive Adams-Oliver syndrome. Am J Hum Genet. 2013;92(4):598-604.

16. Cohen I, Silberstein E, Perez $Y$, et al. Autosomal recessive Adams-Oliver syndrome caused by homozygous mutation in EOGT, encoding an EGF domain-specific O-GlcNAc transferase. Eur J Hum Genet. 2014;22(3):374-378.

17. Stittrich AB, Lehman A, Bodian DL, et al. Mutations in NOTCH1 cause Adams-Oliver syndrome. Am J Hum Genet. 2014;95(3):275-284.

18. Meester JA, Southgate L, Stittrich AB, et al. Heterozygous loss-of-function mutations in DLL4 cause Adams-Oliver syndrome. Am J Hum Genet. 2015;97(3):475-482.

19. Tcherkezian J, Triki I, Stenne R, et al. The human orthologue of CdGAP is a phosphoprotein and a GTPase-activating protein for Cdc42 and Rac1 but not RhoA. Biol Cell. 2006;98(8):445-456.

20. LaLonde DP, Grubinger M, Lamarche-Vane N, Turner CE. CdGAP associates with actopaxin to regulate integrin-dependent changes in cell morphology and motility. Curr Biol. 2006;16(14):1375-1385.

21. Miyamoto Y, Yamauchi J, Sanbe A, Tanoue A. Dock6, a Dock-C subfamily guanine nucleotide exchanger, has the dual specificity for Rac1 and Cdc42 and regulates neurite outgrowth. Exp Cell Res. 2007;313(4):791-804.

22. Wormer $D$, Deakin NO, Turner CE. CdGAP regulates cell migration and adhesion dynamics in two-and three-dimensional matrix environments. Cytoskeleton (Hoboken). 2012;69(9):644-658.

23. Caron C, DeGeer J, Fournier P, et al. CdGAP/ARHGAP31, a Cdc42/Rac1 GTPase regulator, is critical for vascular development and VEGF-mediated angiogenesis. Sci Rep. 2016;6:27485. 
* This manuscript describes the generation and characterisation of an ARHGAP31-deficient mouse and demonstrates a novel role for Arhgap31 in vascular development and angiogenesis, providing support for a vasculogenic origin to AOS pathogenesis.

24. Swartz EN, Sanatani S, Sandor GG, Schreiber RA. Vascular abnormalities in Adams-Oliver syndrome: cause or effect? Am J Med Genet. 1999;82(1):49-52.

25. Cerikan B, Shaheen R, Colo GP, et al. Cell-intrinsic adaptation arising from chronic ablation of a key Rho GTPase regulator. Dev Cell. 2016;39(1):28-43.

26. Cerikan B, Schiebel E. Mechanism of cell-intrinsic adaptation to Adams-Oliver Syndrome gene DOCK6 disruption highlights ubiquitin-like modifier ISG15 as a regulator of RHO GTPases. Small GTPases. 2017 [Epub ahead of print] doi: 10.1080/21541248.2017.1297882

27. Kim K, Yang DK, Kim S, Kang H. miR-142-3p is a regulator of the TGFß-mediated vascular smooth muscle cell phenotype. J Cell Biochem. 2015;116(10):2325-2333.

* This study demonstrates that inhibition of cell migration through DOCK6 down-regulation regulates the TGFB-mediated contractile phenotype of vascular smooth muscle cells.

28. He Y, Northey JJ, Pelletier A, et al. The Cdc42/Rac1 regulator CdGAP is a novel E-cadherin transcriptional co-repressor with Zeb2 in breast cancer. Oncogene. 2017;36(24):3490-3503.

** This article reveals a novel role for ARHGAP31 in the transcriptional regulation of genes in the Notch signalling pathway, supporting a potential link between Rho GTPase activity and Notch pathway regulation in AOS.

29. Ogawa M, Sawaguchi S, Kawai T, et al. Impaired O-linked N-acetylglucosaminylation in the endoplasmic reticulum by mutated epidermal growth factor (EGF) domain-specific O-linked $\mathrm{N}$ acetylglucosamine transferase found in Adams-Oliver syndrome. J Biol Chem. 2015;290(4):2137-2149.

30. Sawaguchi S, Varshney S, Ogawa M, et al. O-GIcNAc on NOTCH1 EGF repeats regulates ligand induced Notch signaling and vascular development in mammals. Elife 2017;6:e24419.

* An investigation of the effects of EOGT loss-of-function on Notch signalling using in vitro assays and Eogt knockout mice, which provides a potential mechanism for EOGT regulation of DLL4-mediated Notch signalling in AOS.

31. MLPA $^{\oplus}$ : An Introduction. Technology: Why Use MLPA ${ }^{\circledR}$ ? MRC-Holland, 2018. Available at: https://www.mlpa.com/WebForms/WebFormMain.aspx?Tag=_hSAvFINWhkPMYt9ZIZdCzsa02M7gmANJd4IBnGypB9MG6kYS4HSWA.. [Last accessed 28 November 2018]

32. Shamseldin HE, Anazi S, Wakil SM, et al. Novel copy number variants and major limb reduction malformation: Report of three cases. Am J Med Genet A. 2016;170A(5):1245-1250.

33. Blanco R, Gerhardt H. VEGF and Notch in tip and stalk cell selection. Cold Spring Harb Perspect Med. 2013;3(1):a006569. 


\section{Table legends:}

Table 1. Diagnostic criteria and categories in Adams-Oliver syndrome Table outlining the clinical criteria compatible with a diagnosis of AOS (diagnostic categories 1a, 1b and $1 \mathrm{c}$ ) or suggestive of a diagnosis of AOS (categories $2 \mathrm{a}, 2 \mathrm{~b}, 2 \mathrm{c}$ ). In each category, the filled circles (O) represent criteria that are required for the diagnosis. Square symbols ( $\square$ ) indicate multiple additional clinical criteria which may also be present but are not necessary for the diagnosis. Unfilled circles $(O)$ indicate exclusive genetic criteria (i.e. pathogenic variation may only be present in one of the genes within each of diagnostic criteria $v$ and vi) which are not necessary for the diagnosis. The minus sign (-) represents criteria which should be absent. ACC: aplasia cutis congenita (with or without an underlying skull defect); CHD: congenital heart defect; CMTC: cutis marmorata telangiectatica congenita; CNS: central nervous system (includes developmental delay); TTLD: terminal transverse limb defects (NB: this covers the full range from hypoplastic nails to hemimelia). *for diagnostic category $2 c$, the affected first-degree relative should have at least TTLD and/or ACC.

Table 2. AOS genes and their phenotypic associations

The distribution of identified mutations is shown as a proportion of all reported AOS cases in the literature and of all independent (unrelated) mutation carriers. The breakdown of phenotypes associated with variants in each gene are detailed as a percentage of all mutation carriers, including related family members. ${ }^{*}$ CNS abnormalities do not include microcephaly, developmental delay or seizures. 
Table 1. Diagnostic criteria and categories in Adams-Oliver syndrome

\begin{tabular}{|c|c|c|c|c|c|c|c|c|c|c|c|c|c|c|}
\hline \multirow{2}{*}{\multicolumn{2}{|c|}{$\begin{array}{r}\begin{array}{r}\text { Diagnostic } \\
\text { criteria: }\end{array} \\
\text { Diagnostic } \\
\text { categories }\end{array}$}} & \multicolumn{2}{|c|}{$\begin{array}{l}\text { i. Hallmark } \\
\text { features }\end{array}$} & \multirow{2}{*}{ 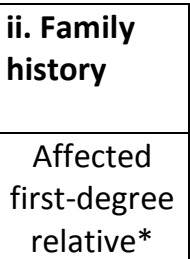 } & \multicolumn{3}{|c|}{$\begin{array}{l}\text { iii. Cardiovascular } \\
\text { defects }\end{array}$} & \multirow{2}{*}{\begin{tabular}{|c|}
$\begin{array}{l}\text { iv. Central nervous } \\
\text { system defects }\end{array}$ \\
$\begin{array}{c}\text { CNS deficiency } \\
\text { (+/- ocular } \\
\text { abnormality) }\end{array}$
\end{tabular}} & \multicolumn{4}{|c|}{$\begin{array}{l}\text { v. Heterozygous variation in an } \\
\text { autosomal dominant AOS gene }\end{array}$} & \multicolumn{2}{|c|}{$\begin{array}{l}\text { vi. Homozygous or compound } \\
\text { heterozygous variation in an } \\
\text { autosomal recessive AOS gene }\end{array}$} \\
\hline & & TTLD & $\begin{array}{l}\text { Scalp } \\
\text { ACC }\end{array}$ & & $\mathrm{CHD}$ & CMTC & $\begin{array}{l}\text { Other } \\
\text { vascular } \\
\text { anomaly }\end{array}$ & & ARHGAP31 & DLL4 & NOTCH1 & $R B P J$ & DOCK6 & $E O G T$ \\
\hline \multirow{15}{*}{ 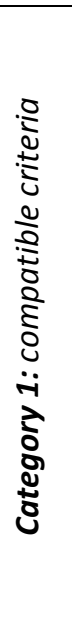 } & $1 a$ & O & O & $\square$ & $\square$ & $\square$ & $\square$ & $\square$ & $\mathrm{O}$ & $\mathrm{O}$ & $\mathrm{O}$ & $\mathrm{O}$ & $\mathrm{O}$ & $\mathrm{O}$ \\
\hline & \multirow[t]{2}{*}{$1 b$} & 0 & $\square$ & 0 & $\square$ & $\square$ & $\square$ & $\square$ & $\mathrm{O}$ & $\mathrm{O}$ & $\mathrm{O}$ & $\mathrm{O}$ & $\mathrm{O}$ & $\mathrm{O}$ \\
\hline & & $\square$ & 0 & 0 & $\square$ & $\square$ & $\square$ & $\square$ & $\mathrm{O}$ & $\mathrm{O}$ & $\mathrm{O}$ & $\mathrm{O}$ & $\mathrm{O}$ & $\mathrm{O}$ \\
\hline & \multirow[t]{12}{*}{$1 \mathrm{c}$} & 0 & $\square$ & $\square$ & $\square$ & $\square$ & $\square$ & $\square$ & 0 & - & - & - & - & - \\
\hline & & 0 & $\square$ & $\square$ & $\square$ & $\square$ & $\square$ & $\square$ & - & 0 & - & - & - & - \\
\hline & & 0 & $\square$ & $\square$ & $\square$ & $\square$ & $\square$ & $\square$ & - & - & 0 & - & - & - \\
\hline & & 0 & $\square$ & $\square$ & $\square$ & $\square$ & $\square$ & $\square$ & - & - & - & 0 & - & - \\
\hline & & 0 & $\square$ & $\square$ & $\square$ & $\square$ & $\square$ & $\square$ & - & - & - & - & 0 & - \\
\hline & & 0 & $\square$ & $\square$ & $\square$ & $\square$ & $\square$ & $\square$ & - & - & - & - & - & 0 \\
\hline & & $\square$ & 0 & $\square$ & $\square$ & $\square$ & $\square$ & $\square$ & 0 & - & - & - & - & - \\
\hline & & $\square$ & 0 & $\square$ & $\square$ & $\square$ & $\square$ & $\square$ & - & 0 & - & - & - & - \\
\hline & & $\square$ & 0 & $\square$ & $\square$ & $\square$ & $\square$ & $\square$ & - & - & 0 & - & - & - \\
\hline & & $\square$ & 0 & $\square$ & $\square$ & $\square$ & $\square$ & $\square$ & - & - & - & 0 & - & - \\
\hline & & $\square$ & 0 & $\square$ & $\square$ & $\square$ & $\square$ & $\square$ & - & - & - & - & 0 & - \\
\hline & & $\square$ & 0 & $\square$ & $\square$ & $\square$ & $\square$ & $\square$ & - & - & - & - & - & 0 \\
\hline \multirow{12}{*}{ 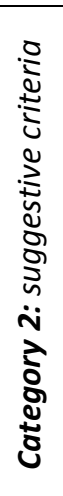 } & \multirow[t]{6}{*}{$2 a$} & 0 & $\square$ & $\square$ & 0 & $\square$ & $\square$ & $\square$ & 0 & 0 & 0 & 0 & 0 & 0 \\
\hline & & 0 & $\square$ & $\square$ & $\square$ & 0 & $\square$ & $\square$ & 0 & 0 & 0 & 0 & 0 & 0 \\
\hline & & 0 & $\square$ & $\square$ & $\square$ & $\square$ & 0 & $\square$ & 0 & 0 & 0 & 0 & 0 & $\bigcirc$ \\
\hline & & $\square$ & 0 & $\square$ & 0 & $\square$ & $\square$ & $\square$ & 0 & 0 & 0 & 0 & 0 & 0 \\
\hline & & $\square$ & 0 & $\square$ & $\square$ & 0 & $\square$ & $\square$ & 0 & 0 & 0 & 0 & 0 & 0 \\
\hline & & $\square$ & 0 & $\square$ & $\square$ & $\square$ & 0 & $\square$ & 0 & 0 & 0 & 0 & 0 & 0 \\
\hline & \multirow[t]{2}{*}{$2 b$} & 0 & $\square$ & $\square$ & $\square$ & $\square$ & $\square$ & 0 & 0 & 0 & 0 & 0 & 0 & 0 \\
\hline & & $\square$ & 0 & $\square$ & $\square$ & $\square$ & $\square$ & 0 & 0 & 0 & 0 & 0 & 0 & 0 \\
\hline & \multirow[t]{4}{*}{$2 c$} & $\square$ & $\square$ & 0 & 0 & $\square$ & $\square$ & $\square$ & 0 & 0 & 0 & 0 & 0 & 0 \\
\hline & & $\square$ & $\square$ & 0 & $\square$ & 0 & $\square$ & $\square$ & 0 & 0 & 0 & 0 & 0 & 0 \\
\hline & & $\square$ & $\square$ & 0 & $\square$ & $\square$ & 0 & $\square$ & 0 & 0 & 0 & 0 & 0 & 0 \\
\hline & & $\square$ & $\square$ & 0 & $\square$ & $\square$ & $\square$ & 0 & 0 & 0 & 0 & 0 & 0 & 0 \\
\hline
\end{tabular}


Table 2. AOS genes and their phenotypic associations

\begin{tabular}{|c|c|c|c|c|c|c|c|}
\hline \multirow{2}{*}{$\begin{array}{l}\text { Inheritance } \\
\text { Gene name }\end{array}$} & \multicolumn{4}{|c|}{ Autosomal dominant } & \multicolumn{2}{|c|}{ Autosomal recessive } & \multirow[t]{3}{*}{ Total } \\
\hline & ARHGAP31 & DLL4 & NOTCH1 & $R B P J$ & DOCK6 & $E O G T$ & \\
\hline Chromosomal locus & $3 q 13.32-q 13.33$ & $15 q 15.1$ & $9 q 34.3$ & $4 p 15.2$ & $19 p 13.2$ & $3 p 14.1$ & \\
\hline Number of independent mutations reported & 6 & 15 & 27 & 6 & 19 & $\begin{array}{l}17 \text { (of which } 9 \text { are a } \\
\text { recurrent founder mutation) }\end{array}$ & 90 \\
\hline$\%$ of all reported AOS families ( $n=249)$ & $2.4 \%$ & $6.0 \%$ & $10.8 \%$ & $2.4 \%$ & $7.6 \%$ & $6.8 \%$ & $36 \%$ \\
\hline$\%$ of independent mutation carriers $(n=90)$ & $6.7 \%$ & $16.7 \%$ & $30.0 \%$ & $6.7 \%$ & $21.1 \%$ & $18.9 \%$ & $100 \%$ \\
\hline Percentage of all mutation carriers with: & & & & & & & $\begin{array}{l}\% \text { in all reported AOS } \\
\text { cases }(n=434)\end{array}$ \\
\hline Scalp ACC & 32 & 92 & 62 & 90 & 95 & 96 & 81 \\
\hline TTLD or other limb defect & 82 & 50 & 60 & 100 & 100 & $\begin{array}{l}70 \text { (of which } 55 \% \text { have } \\
\text { cutaneous syndactyly) }\end{array}$ & 81 \\
\hline Congenital heart defect & 0 & 21 & 36 & 30 & 33 & 11 & 23 \\
\hline Cutis marmorata telangiectatica congenita & 0 & 25 & 23 & 10 & 10 & 11 & 20 \\
\hline Other vascular anomaly & 0 & 8 & 23 & 10 & 5 & 22 & 16 \\
\hline Central nervous system abnormality* & 0 & 13 & 9 & 10 & 81 & 11 & 19 \\
\hline Microcephaly & 0 & 8 & 0 & 30 & 57 & 0 & 11 \\
\hline Developmental delay & 0 & 8 & 9 & 10 & 57 & 7 & 10 \\
\hline Seizures/epilepsy & 0 & 4 & 2 & 0 & 57 & 4 & 8 \\
\hline Structural eye anomaly & 0 & 4 & 0 & 0 & 57 & 0 & 6 \\
\hline
\end{tabular}

